\title{
Generation and annotation of the DNA sequences of human chromosomes 2 and 4
}

LaDeana W. Hillier ${ }^{1}$, Tina A. Graves ${ }^{1}$, Robert S. Fulton ${ }^{1}$, Lucinda A. Fulton ${ }^{1}$, Kymberlie H. Pepin ${ }^{1}$, Patrick Minx ${ }^{1}$, Caryn Wagner-McPherson ${ }^{1}$, Dan Layman $^{1}$, Kristine Wylie ${ }^{1}$, Mandeep Sekhon ${ }^{1}$, Michael C. Becker ${ }^{1}$, Ginger A. Fewell ${ }^{1}$, Kimberly D. Delehaunty ${ }^{1}$, Tracie L. Miner ${ }^{1}$, William E. Nash ${ }^{1}$, Colin Kremitzki ${ }^{1}$, Lachlan Oddy ${ }^{1}$, Hui Du ${ }^{1}$, Hui Sun ${ }^{1}$, Holland Bradshaw-Cordum ${ }^{1}$, Johar Ali ${ }^{1}$, Jason Carter ${ }^{1}$, Matt Cordes ${ }^{1}$, Anthony Harris ${ }^{1}$, Amber Isak ${ }^{1}$, Andrew van Brunt ${ }^{1}$, Christine Nguyen ${ }^{1}$, Feiyu Du ${ }^{1}$, Laura Courtney ${ }^{1}$, Joelle Kalicki ${ }^{1}$, Philip Ozersky ${ }^{1}$, Scott Abbott ${ }^{1}$, Jon Armstrong ${ }^{1}$, Edward A. Belter ${ }^{1}$, Lauren Caruso ${ }^{1}$, Maria Cedroni ${ }^{1}$, Marc Cotton ${ }^{1}$, Teresa Davidson $^{1}$, Anu Desai ${ }^{1}$, Glendoria Elliott ${ }^{1}$, Thomas Erb ${ }^{1}$, Catrina Fronick ${ }^{1}$, Tony Gaige ${ }^{1}$, William Haakenson ${ }^{1}$, Krista Haglund ${ }^{1}$, Andrea Holmes ${ }^{1}$, Richard Harkins ${ }^{1}$, Kyung Kim ${ }^{1}$, Scott S. Kruchowski ${ }^{1}$, Cynthia Madsen Strong ${ }^{1}$, Neenu Grewal ${ }^{1}$, Ernest Goyea ${ }^{1}$, Shunfang Hou ${ }^{1}$, Andrew Levy ${ }^{1}$, Scott Martinka ${ }^{1}$, Kelly Mead ${ }^{1}$, Michael D. McLellan' ${ }^{1}$, Rick Meyer ${ }^{1}$, Jennifer Randall-Maher ${ }^{1}$, Chad Tomlinson ${ }^{1}$, Sara Dauphin-Kohlberg ${ }^{1}$, Amy Kozlowicz-Reilly ${ }^{1}$, Neha Shah ${ }^{1}$, Sharhonda Swearengen-Shahid ${ }^{1}$, Jacqueline Snider ${ }^{1}$, Joseph T. Strong ${ }^{1}$, Johanna Thompson ${ }^{1}$, Martin Yoakum ${ }^{1}$, Shawn Leonard ${ }^{1}$, Charlene Pearman ${ }^{1}$, Lee $\operatorname{Trani}^{1}$, Maxim Radionenko ${ }^{1}$, Jason E. Waligorski ${ }^{1}$, Chunyan Wang ${ }^{1}$, Susan M. Rock ${ }^{1}$, Aye-Mon Tin-Wollam ${ }^{1}$, Rachel Maupin ${ }^{1}$, Phil Latreille ${ }^{1}$, Michael C. Wendl ${ }^{1}$, Shiaw-Pyng Yang ${ }^{1}$, Craig Pohl ${ }^{1}$, John W. Wallis ${ }^{1}$, John Spieth ${ }^{1}$, Tamberlyn A. Bieri ${ }^{1}$, Nicolas Berkowicz ${ }^{1}$, Joanne O. Nelson ${ }^{1}$, John Osborne ${ }^{1}$, Li Ding ${ }^{1}$, Rekha Meyer ${ }^{1}$, Aniko Sabo ${ }^{1}$, Yoram Shotland ${ }^{1}$, Prashant Sinha ${ }^{1}$, Patricia E. Wohldmann ${ }^{1}$, Lisa L. Cook ${ }^{1}$, Matthew T. Hickenbotham ${ }^{1}$, James Eldred ${ }^{1}$, Donald Williams ${ }^{1}$, Thomas A. Jones ${ }^{2}$, Xinwei She ${ }^{3}$, Francesca D. Ciccarelli ${ }^{4}$, Elisa Izaurralde ${ }^{4}$, James Taylor ${ }^{5}$, Jeremy Schmutz ${ }^{6}$, Richard M. Myers ${ }^{6}$, David R. Cox ${ }^{6 \star}$, Xiaoqiu Huang ${ }^{7}$, John D. McPherson ${ }^{1}$, Elaine R. Mardis ${ }^{1}$, Sandra W. Clifton ${ }^{1}$, Wesley C. Warren ${ }^{1}$, Asif T. Chinwalla ${ }^{1}$, Sean R. Eddy ${ }^{2}$, Marco A. Marra ${ }^{1}$, Ivan Ovcharenko ${ }^{8}$, Terrence S. Furey ${ }^{9}$, Webb Miller ${ }^{5}$, Evan E. Eichler ${ }^{3}$, Peer Bork ${ }^{4}$, Mikita Suyama ${ }^{4}$, David Torrents ${ }^{4}$, Robert H. Waterston ${ }^{1}$ \& Richard K. Wilson $^{1}$

${ }^{1}$ Genome Sequencing Center, Washington University School of Medicine, Campus Box 8501, 4444 Forest Park Avenue, St. Louis, Missouri 63108, USA

${ }^{2}$ Howard Hughes Medical Institute and Department of Genetics, Washington University School of Medicine, St. Louis, Missouri 63110, USA

${ }^{3}$ Genome Sciences, University of Washington School of Medicine, Seattle, Washington 98195, USA

${ }^{4}$ EMBL, Meyerhofstrasse 1, Heidelberg 69117, Germany

${ }^{5}$ Center for Comparative Genomics and Bioinformatics, Departments of Biology and Computer Science, Pennsylvania State University, University Park, Pennsylvania 16802, USA

${ }^{6}$ Stanford Human Genome Center, Department of Genetics, Stanford University School of Medicine, Stanford, California 94305, USA

${ }^{7}$ Department of Computer Science, Iowa State University, Ames, Iowa 50011-1040, USA

${ }^{8}$ EEBI Division and Genome Biology Division, Lawrence Livermore National Laboratory, Livermore, California 94550, USA

${ }^{9}$ Center for Biomolecular Science and Engineering, University of California, Santa Cruz, California 95064, USA

* Present addresses: Perlegen Sciences Inc., 2021 Stierlin Court, Mountain View, California 94943, USA (D.R.C); Baylor College of Medicine, 1 Baylor Plaza, Human Genome Sequencing Center, N1519, Houston, Texas 77030, USA (J.D.M.); Genome Sciences Centre, British Columbia Cancer Agency, 600 West 10th Avenue, Room 3427, Vancouver, British Columbia V5Z 4E6, Canada (M.A.M.); Department of Genome Sciences, Box 357730, University of Washington, 1705 NE Pacific Street, Seattle, Washington 98195-7730, USA (R.H.W.).

Human chromosome 2 is unique to the human lineage in being the product of a head-to-head fusion of two intermediate-sized ancestral chromosomes. Chromosome 4 has received attention primarily related to the search for the Huntington's disease gene, but also for genes associated with Wolf-Hirschhorn syndrome, polycystic kidney disease and a form of muscular dystrophy. Here we present approximately 237 million base pairs of sequence for chromosome 2, and 186 million base pairs for chromosome 4 , representing more than $99.6 \%$ of their euchromatic sequences. Our initial analyses have identified 1,346 protein-coding genes and 1,239 pseudogenes on chromosome 2, and 796 protein-coding genes and 778 pseudogenes on chromosome 4 . Extensive analyses confirm the underlying construction of the sequence, and expand our understanding of the structure and evolution of mammalian chromosomes, including gene deserts, segmental duplications and highly variant regions.

Less than 50 years after the human diploid number was established, the reference human genome sequence was announced ${ }^{1}$. Detailed accounts of the sequences of individual chromosomes are now providing great insights into genomic structure and evolution. Here we present our analysis of the sequence of human chromosomes 2 and 4 . For chromosome 2, we analyse the region containing the ancestral chromosome fusion event ${ }^{2}$ and describe possible mechanisms for the inactivation of the vestigial centromere. For chromosome 4, we discover some regions with the lowest and highest $(\mathrm{G}+\mathrm{C})$ content in the human genome, as well as the putative largest 'gene deserts'. Analyses of highly variant regions found on these chromosomes have also allowed us to investigate their origins.
Generation of the chromosome sequences

Chromosomes 2 and 4 were sequenced using a clone-by-clone shotgun sequencing strategy ${ }^{3}$ supported by the bacterial artificial chromosome (BAC)-based whole genome physical map ${ }^{4}$. The quality of the sequences was determined to exceed the $99.99 \%$ accuracy standard ${ }^{1,5,6}$. On both chromosomes, sequences extend into the centromere and reach the p-arm telomere ${ }^{7}$ (Supplementary Table 1). Attempts were made to close all remaining gaps ${ }^{8}$ (Supplementary Methods), with an emphasis on using newly available fosmid libraries. Seventy-six clones were selected from the fosmid end placements ${ }^{1}$, which added $\sim 500 \mathrm{~kb}$ of sequence (included in release hg17/build35). On the basis of size estimates of remaining 
gaps, the available sequence represents more than $99.6 \%$ of the total euchromatic sequence.

The integrity of the underlying clone sequences and their assembly into chromosomes were verified by comparisons of an in silico digest of each finished sequence to the restriction digests of the clone DNA, and comparison of the full assembly sequence against the underlying fingerprint data. In this way, we directly confirmed more than $99.99 \%$ of the testable bands. In addition, by examining the placements of BAC, fosmid and plasmid ${ }^{9}$ paired end sequences, and by comparing the order of $\mathrm{BAC}$ end placements to the order of the BACs within the fingerprint map ${ }^{4}$, we confirmed the overall consistency of the map, sequence and assembly (Supplementary Methods).

\section{Comparison to physical and genetic maps}

We have used the Genethon ${ }^{10}$, Marshfield ${ }^{11}$ and deCODE ${ }^{12}$ microsatellite-based maps, GeneMap $99^{13}$ and $\mathrm{TNG}^{14}$ radiation hybrid maps, the Whitehead yeast artificial chromosome (YAC)-based map and the BAC physical map ${ }^{4}$ to evaluate the completeness and order of assembled sequences for chromosomes 2 and 4 . Only a small number $(<1 \%)$ of sequence-tagged sites (STSs) were not identified in the existing sequence; these included STSs from repetitive sequences, sequence polymorphisms and regions within known sequence gaps.

To evaluate the assembly of the sequence, the chromosomal sequence positions of the deCODE STSs were plotted relative to their established map positions (Supplementary Fig. 1). When examining local ordering of the deCODE marker set (the average spacing of which is $\sim 1$ per $600 \mathrm{~kb}$ ), there were no long-range disagreements, and a local inversion of marker pairs was found in only one of the placements. Taken together, these data provide strong support for the presented sequences of chromosomes 2 and 4 .

\section{General features}

We analysed the chromosome 2 and 4 sequences for interspersed repeat content (Supplementary Table 2), $(\mathrm{G}+\mathrm{C}$ ) content, recombination and the presence of $\mathrm{CpG}$ islands. For both chromosomes, the short interspersed nucleotide element (SINE) content is lower than the autosomal average. The long interspersed nucleotide element (LINE) content is higher than average for both chromosomes, with chromosome 4 containing the highest percentage across all autosomes.

The $(\mathrm{G}+\mathrm{C})$ content of chromosomes $2(40.2 \%)$ and $4(38.2 \%)$ is lower than that of the genome as a whole $(41 \%)$. On chromosome 2 , the lowest $(\mathrm{G}+\mathrm{C})$ content $(29.9 \%)$ is found in a $40-\mathrm{kb}$ region containing no known genes. Over $19 \%$ of chromosome 4 has a $(\mathrm{G}+\mathrm{C})$ content of less than $35 \%$, compared with $9 \%$ in the genome as a whole. However, one of the highest $(\mathrm{G}+\mathrm{C})$ content windows in the genome $(72.7 \%)$ is also found on chromosome 4.

We identified 1,662 CpG islands in chromosome 2 (7 per Mb), and 1,004 CpG islands in chromosome 4 (5.4 per Mb), each with an average length of $\sim 800 \mathrm{bp}$. Chromosome 4 has the lowest density of predicted $\mathrm{CpG}$ islands of any human chromosome. For the gene sets presented below, 67\% (chromosome 2) and 64\% (chromosome 4) had overlapping CpG islands, consistent with other estimates ${ }^{15,16}$.
Chromosomes 2 and 4 have the lowest average recombination rate of any of the chromosomes (1.09 compared with the genome average of 1.31). Each chromosome contains large regions with little or no recombination (Supplementary Table 3). For chromosome 2, these regions include the candidate tumour suppressor gene $L R P 1 B^{17}$, which spans $500 \mathrm{~kb}$, the ZAP70 gene ${ }^{18}$ and TTN, the longest coding sequence in the human genome, spanning $280 \mathrm{~kb}$ and encoding a $2,993 \mathrm{kDa}$ protein. On chromosome 4 , a recombination cold spot was identified at $\sim 42 \mathrm{Mb}$, the location of the paired-like homeobox $2 \mathrm{~b}$ gene $(\mathrm{PHOX} 2 \mathrm{~B})$. As expected, the primary hot spots are located near the telomeres, where recombination rates are five- to tenfold higher than average.

\section{Protein-coding genes: known genes}

Creation of protein-coding gene indices for chromosomes 2 and 4 exploited both the increasing number of available human messenger RNAs and the extensive orthology with mouse (defined for $94 \%$ of chromosome 2 and $97 \%$ of chromosome 4), in a hierarchical approach similar to that used for human chromosome 7 (ref. 8). First, a collection of 1,448 (chromosome 2) and 820 (chromosome 4) human mRNAs from RefSeq ${ }^{19}$ and the Mammalian Gene Collection $(\mathrm{MGC})^{20}$ were assigned to the sequence and manually curated, resulting in 1,032 non-overlapping mRNAs on chromosome 2 and 633 on chromosome 4 . On the basis of alignments with known mRNAs, $25 \%$ of the known genes on these chromosomes have alternative splice forms. However, we estimate that this percentage rises to as high as $85 \%$ on the basis of gene structure prediction using expressed-sequence-tag (EST) data and all vertebrate mRNAs with an average number of 5 transcripts per gene. Of the known genes, $89 \%$ of those on chromosome 2 and $86 \%$ of those on chromosome 4 had an associated poly(A) signal. All known mRNAs previously mapped to these chromosomes were successfully identified, confirming the completeness of the available sequence.

Alignment of the human mRNA set (RefSeq and MGC) against the genome revealed both insights and potentially confounding artefacts. For example, detailed examination identified a set of 36 genes (Supplementary Table 4) predicted from the known mRNAs that had no match at all to the mouse or rat genomes or their protein sets; 26 of these had no similarity to the non-redundant protein database. Sixteen of the 26 genes were single-exon genes, and might be fortuitous open reading frames (ORFs) in untranslated regions (UTRs). Eleven of these single-exon genes had ORFs of more than $300 \mathrm{bp}$ (the average across the 16 single-exon genes was $350 \mathrm{bp}$ ). The remaining ten were multi-exon genes. To test whether these were novel human genes, we obtained sequences from other primates for six of the ten multi-exon genes. For five out of six genes, there was an ORF through the re-sequenced exon in each of the equivalent primate genes. In the sixth gene (Genbank accession number NM_153031, the representative entry for 3 underlying mRNAs also supported by EST sequences from human testis and brain libraries), only the chimp sequence had an ORF throughout the coding exon. The $K_{\mathrm{a}} / K_{\mathrm{s}}$ ratio (the ratio of non-synonymous $\left(K_{\mathrm{a}}\right)$ to synonymous $\left(K_{\mathrm{s}}\right)$ nucleotide substitution rates) was 0.342 , suggestive of purifying selection.

Table 1 Sequence and frequency of polymorphic mRNAs

\begin{tabular}{|c|c|c|c|c|c|c|c|}
\hline \multirow[t]{2}{*}{ Gene ID } & \multirow[t]{2}{*}{ Gene } & \multirow[t]{2}{*}{ BAC sequence } & \multirow[t]{2}{*}{ mRNA sequence } & \multicolumn{4}{|c|}{ Numbers of individuals with genotype (out of 24)† } \\
\hline & & & & $\mathrm{BAC} / \mathrm{BAC}$ & $\mathrm{BAC} / \mathrm{mRNA}$ & mRNA/mRNA & $\mathrm{n} / \mathrm{a}$ \\
\hline PMS1 & post-meiotic segregration increase 1 & AAA*GTTACT & AAAAGTTACT & 14 & 8 & 1 & 1 \\
\hline PASK & PAS domain-containing serine/threonine kinase & $\mathrm{C} \pi \| \Pi^{*} \mathrm{GCAG}$ & CTIIITGCAG & 18 & 5 & 0 & 1 \\
\hline AUP1 & ancient ubiquitous protein 1 & GTCAG*TITाT & GTCAGTIIIT & 7 & 11 & 2 & 4 \\
\hline TSARG2 & testis and spermatogenesis cell related protein 2 & ATAGAGAAAT & ATA*AAT & 20 & 2 & 0 & 2 \\
\hline
\end{tabular}

An asterisk in the DNA sequence indicates the position of a nucleotide difference between the corresponding BAC and mRNA sequences.

†BAC/BAC, number of individuals for whom the sequence agrees with the sequence obtained from the genomic sequencing effort; BAC/mRNA, number of heterozygotes; mRNA/mRNA, number of individuals for whom the sequence agrees with the mRNA; $n / a$, number of individuals for whom the sequence of the PCR product could not be obtained. See Supplementary Table 5 for additional details. 
We also investigated 81 genes for which the genomic sequence differed from the corresponding mRNA, causing alterations to or truncation of the protein product predicted from the genome sequence. To determine the nature of these differences, we used polymerase chain reaction (PCR) to re-sequence each gene in a panel of 24 ethnically diverse individuals ${ }^{21}$ and in underlying BAC clones. Eight out of the 81 genes were found to contain errors in the genomic sequence. For 69 of the 81 genes, the sequence obtained from the 24 individuals agreed with the sequence of the original BAC clone, suggesting errors in the mRNA sequence or rare polymorphisms. Of these, 54 were single base-insertion or -deletion errors in the mRNA that shifted or truncated the reading frame in the complementary DNA relative to the genomic sequence. The other 15 mRNAs had multiple base-insertion or -deletion differences, such that the frame was eventually restored. In these cases, comparison with a related mouse gene sequence confirmed the genomic translation, typically with a more conserved match between the mouse and human genome sequences than with the corresponding human mRNA.

Four genes were determined to be polymorphic for gene disruptions in the 24 genomic samples (Table 1 and Supplementary Table 5), none of which had previously been annotated in public databases. For example, a single base-deletion event in an alternative $3^{\prime}$ exon was observed in the PMS1 gene, resulting in a change and extension of the ORF. Some cases of hereditary nonpolyposis colorectal cancer are associated with mutations in this gene $\mathrm{e}^{22}$. In a second example, a polymorphic frameshift was observed in the final exon of the PASK mRNA, leading to a longer ORF in genomic translation. However, the similarity to the orthologous mouse gene does not extend past the stop codon of the mRNA. In a third case, a polymorphic single base deletion identified in the longest isoform of AUP1 resulted in an early, in-frame stop codon, which truncated the protein at one-third of its normal length (149 out of 476 residues). Finally, an insertion of $4 \mathrm{bp}$ was found in the genomic sequence of TSARG2 (also known as SPATA4) relative to the RefSeq mRNA, causing a frameshift and early truncation of the protein. Similarity between the orthologous mouse protein and genomic sequence extends through the region of the frameshift.

In addition, we detected 41 potential polymorphic frameshifts by aligning genomic coding regions of chromosomes 2 and 4 (confirmed by mRNAs) to random genomic shotgun data ${ }^{9}$ and examining high-quality insertion and deletion differences. When comparing these regions to the orthologous chimpanzee sequence (Chimpanzee Genome Sequencing Consortium (GSC), unpublished data), the orthologous chimpanzee sequence agreed with the human BAC sequence in 37 instances. This confirms the genome sequence but does not rule out true polymorphism. For the four remaining frameshifts, confirmation with the chimpanzee sequence served to eliminate error in the random reads as a cause of the frameshift, indicating that the genome carries the derived allele. Thus, using the available chimpanzee sequence, we have identified

\begin{tabular}{|c|c|c|c|c|c|c|}
\hline \multicolumn{7}{|c|}{ Table 2 Coverage of predicted and known genes by various data sets } \\
\hline \multirow[t]{2}{*}{ Data set } & \multicolumn{2}{|c|}{$\begin{array}{c}\text { Predicted genes } \\
\text { (\%) }\end{array}$} & \multicolumn{2}{|c|}{$\begin{array}{c}\text { Known genes } \\
(\%)\end{array}$} & \multicolumn{2}{|c|}{ Ratio* } \\
\hline & Chr 2 & Chr 4 & Chr 2 & Chr 4 & Chr 2 & Chr 4 \\
\hline Human ESTs & 69 & 58 & 94 & 93 & 0.73 & 0.62 \\
\hline Non-mammalian† & 69 & 79 & 95 & 92 & 0.73 & 0.86 \\
\hline Gallus gallus & 64 & 76 & 92 & 91 & 0.70 & 0.84 \\
\hline Totalł & 89 & 92 & 99 & 99 & 0.90 & 0.93 \\
\hline Pfam & 41 & 48 & 76 & 73 & 0.54 & 0.66 \\
\hline Interpro & 48 & 60 & 82 & 77 & 0.59 & 0.80 \\
\hline
\end{tabular}

Chr, chromosome

${ }^{*}$ Ratio of predicted genes to known genes.

†Protein sets from non-mammalian vertebrate genomes: Fugu rubripes ${ }^{37}$, Tetraodon nigroviridis ${ }^{48}$ Danio rerio (Sanger Centre, unpublished data), and Ciona intestinalis ${ }^{49}$

$\ddagger$ The percentage of genes sharing similarities with human ESTs, non-mammalian vertebrate genomes and/or Gallus gallus. four probable polymorphic gene disruptions not yet annotated in the public databases.

\section{Protein-coding genes: predicted genes}

Predicted genes were identified on chromosomes 2 and 4 using GENEWISE $^{23}$ (which uses protein homologies to seed prediction), and TWINSCAN ${ }^{24}$ (which uses comparative sequence analysis, in this case using the mouse genome sequence). A previous difficulty in using GENEWISE, especially in regions containing duplicated gene clusters, has been definition of the boundaries of the genomic region provided as input. We have alleviated this problem using the program BLAST2GENE ${ }^{25}$ to detect (using homology) independent copies of genes, ranging from single exons to complete copies. The combined output predicted $99.5 \%$ of all known exons and at least part of $99.8 \%$ of the known genes, indicating that for known genes, the combined output is reasonably comprehensive and has high sensitivity.

Using methods similar to those for our analyses of human chromsome 7 (see ref. 8 and Supplementary Methods), we refined the initial predicted gene set. Predicted genes were required to have a highly significant match in the mouse gene set in the orthologous region of the mouse genome (if assigned), and the matching mouse gene was required to have the predicted chromosome 2 or 4 gene as its best match. Redundancy between the sets, within the sets, and with known genes was eliminated, accepting the already known genes, the GENEWISE predictions and then the TWINSCAN predictions, in that order. This yielded 314 predicted genes for chromosome 2 and 163 predicted genes on chromosome 4, bringing the total number of protein-coding genes to 1,346 and 796, respectively.

The pseudogene analysis below confirms that few functional protein-coding genes have been missed. Of the spliced ESTs mapping to chromosomes 2 and 4, 97\% overlap an exon or are within $1 \mathrm{~kb}$ of an initial or terminal exon in the gene set. The nonoverlapping ESTs might represent some coding genes, but are more likely to be other transcription products, including noncoding RNA genes or untranslated fragments of protein-coding genes. A high percentage of the predicted genes can be matched with human ESTs and have similarities with non-mammalian vertebrate protein sets (Table 2). The higher percentages for the known genes (compared with the predicted genes) probably reflect the fact that they are in general more highly expressed and, in many cases, more highly conserved across evolution. Comparisons of gene structure characteristics for known and predicted genes (Table 3) reveal similar numbers of coding bases per gene. Smaller exon counts and gene lengths for the predicted gene set might reflect fragmentation or missing terminal exons. Finally, the pseudogene analysis described below suggests that the protein-coding gene set is relatively free of pseudogenes. We conclude that the combined set of known and predicted protein coding genes is both reasonably comprehensive and free of false predictions.

\section{Pseudogenes}

With the availability of the complete genome sequence for $\mathrm{rat}^{26}$ and mouse $^{27}$, and with the sequences for other mammalian genomes in progress, we have significantly refined our pseudogene catalogues $^{8,26,28}$. For chromosomes 2 and 4, we have further improved

\begin{tabular}{|c|c|c|c|c|c|c|}
\hline & \multicolumn{2}{|c|}{ Predicted genes } & \multicolumn{2}{|c|}{ Known genes } & \multicolumn{2}{|c|}{ Ratio* } \\
\hline & Chr 2 & Chr 4 & Chr 2 & Chr 4 & Chr 2 & Chr 4 \\
\hline Exons per gene & 6.6 & 5.3 & 10.5 & 9.5 & 0.63 & 0.56 \\
\hline Coding bases per gene (bp) & 1,150 & 1,009 & 1,662 & 1,149 & 0.69 & 0.88 \\
\hline Genic bases per gene (kb) & 33.8 & 34.3 & 66.4 & 75.1 & 0.51 & 0.46 \\
\hline
\end{tabular}

${ }^{\star}$ Ratio of predicted genes to known genes. 
our detection and classification algorithms to define a set of 1,239 and 778 intergenic regions considered pseudogenes because they show homology to existing proteins and because nearly all (94 $\pm 3 \%$ and $95 \pm 3 \%$ ) appear to evolve neutrally according to the $K_{\mathrm{a}} / K_{\mathrm{s}}$ ratio test ${ }^{28}$. In contrast, only $5 \pm 3 \%$ of the proteincoding set have a $K_{\mathrm{a}} / K_{\mathrm{s}}$ ratio consistent with neutral evolution.

We distinguished retrotransposed (processed) from segmentally duplicated (non-processed) pseudogenes on the basis of their sequence similarity in orthologous regions of the mouse genome because processed pseudogenes tend to integrate throughout the genome (probably far from their functional paralogues). Out of 230 pseudogenes on chromosomes 2 and 4 with detectable sequence similarity to their mouse orthologous block, 94 appeared to be nonprocessed because they also shared similarity with neighbouring genes. Of the remaining pseudogenes (136), nearly all were shown to have lost at least one intron when compared with their parental gene, and were therefore considered processed pseudogenes. The sequence identity between these processed pseudogenes and their parental human genes is significantly higher $(>10 \%$ difference in nearly all cases) than the sequence identity between the pseudogenes and the orthologous mouse regions. This strongly suggests that the pseudogenes arose independently in the human and mouse lineages, rather than from a common ancestor as recently proposed $^{29}$. Altogether, we defined a set of 1,856 processed and 161 non-processed pseudogenes, consistent with estimates of $\sim 20,000$ pseudogenes across the human genome ${ }^{28}$.

\section{Non-coding RNAs}

We identified non-coding RNA (ncRNA) genes in the chromosome sequences as described for the human genome sequence ${ }^{1}$. With the exception of close sequence homologues and transfer RNAs, ncRNA gene annotation is still largely limited to annotating known gene sequences $^{30}$. Chromosomes 2 and 4 contain $15 \%$ of the unambiguous bases in the human genome and $14 \%$ of the annotated ncRNA pseudogenes ( 863 out of 6,124 ), but only $2 \%$ of the tRNAs and only $5 \%$ of all annotated ncRNA genes (50 out of 1,096). The ncRNA pseudogene annotation includes 163 tRNA pseudogenes, 150 of which were derived from ancient mtDNA integrations into the nuclear genome (nuclear mitochondrial DNAs or NUMTs ${ }^{31}$ ). Previous annotation missed most of these mtDNA-derived pseudogenes, because their sequence divergence is usually too great to recognize them individually. However, here we have identified these pseudogenes by searching for clustering of weak hits in a larger expanse of decayed mtDNA synteny.

\section{Protein index}

We derived an index of predicted protein sequences for human chromosomes 2 and 4, and compared them to the Interpro database $^{32}$ using Interproscan ${ }^{33}$ to predict protein families, domain and repeat families, and sequence motifs. Of the $74 \%$ of proteins that had an Interpro classification, $67 \%$ were multi-domain. Protein kinases are the most highly represented families on the two chromosomes. Gene clusters included the most prevalent protein families in the human genome, the immunoglobulins and zincfinger domain-containing proteins. On chromosome 2, a cluster of 13 genes containing immunoglobulin-like domains (Interpro identifier IPR007110) is found on the p-arm near the centromere, and a cluster of 11 genes containing zinc-finger domains $\left(\mathrm{C}_{2} \mathrm{H}_{2}\right.$

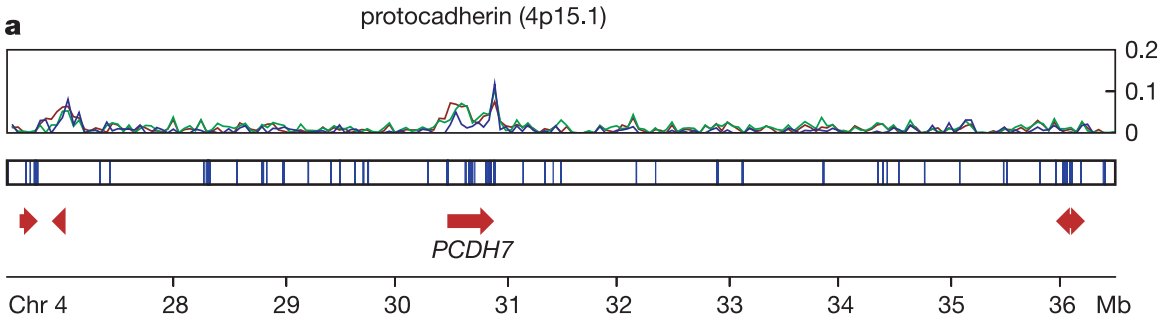

b

$4 q 34.3$

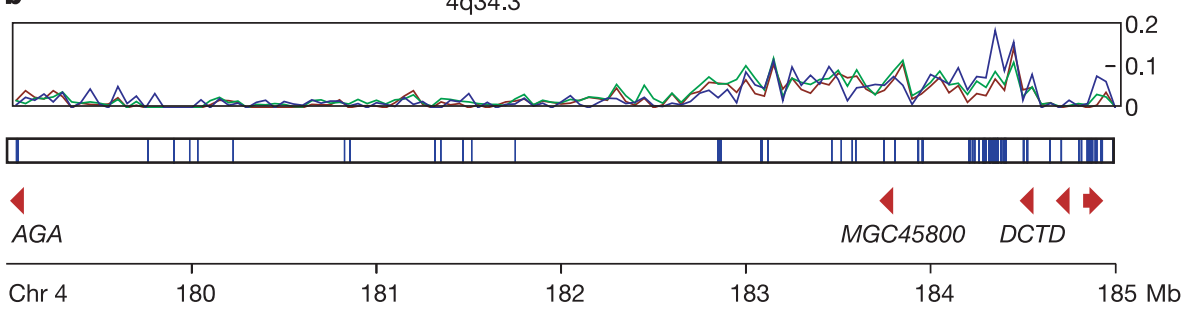

c

ZFHX1B (2q22.3)

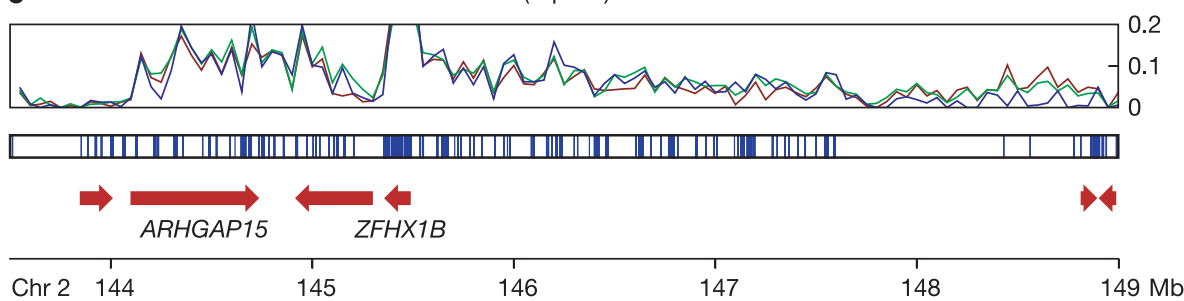

Figure 1 Three gene deserts. Levels of non-coding conservation are plotted in nonoverlapping 50-kb windows between human and dog (green), human and mouse (red), and human and chicken (blue). The second panel in each of a-c shows positions of matches between human and Fugu rubripes (including coding regions) computed by the $B L A T^{50}$ program. Red symbols show the positions and orientations of genes. a, A 10-Mb interval of chromosome 4 centred on the protocadherin gene $P C D H 7$. b, A 6-Mb interval of chromosome 4 containing one of the longest human gene deserts, showing significant enrichment for matches to chicken, mouse and dog. c, A 5.5-Mb interval of chromosome 2 that contains the longest human segment showing high enrichment for non-coding matches with chicken. 
type; IPR007087) is found on the q-arm. On chromosome 4, the largest clusters are grouped near each other on the q-arm: one cluster contains nine genes with UDP-glucosyltransferase domains (IPR002213), and another cluster contains eight small chemokines (of the C-X-C/interleukin-8 subfamily; IPR002473). The Interpro results were used to assign Gene Ontology (GO) $\operatorname{codes}^{34}$ : for chromosomes 2 and $4,63 \%$ and $68 \%$ of proteins were assigned to the global category of molecular function, $51 \%$ and $57 \%$ to biological process, and $34 \%$ and $39 \%$ to cellular components, respectively. The two most frequent specific GO categories for both chromosomes were the cellular components of nucleus (GO:0005634) and membrane (GO:0016020), representing 15\% (chromosome 2) and 19\% (chromosome 4) of the genes with assigned function.

\section{Gene deserts and conserved non-coding sequence}

Gene deserts are a curious feature of vertebrate genomes; they are megabase-size genomic segments devoid of protein-coding genes. The overall architectures of these regions are maintained over long evolutionary distances, sometimes with only very limited sequence conservation $^{35}$. In other cases, the deserts have been found to contain small blocks of highly conserved sequences that regulate flanking genes. Roughly $80 \%$ of human gene deserts occur in $(\mathrm{G}+\mathrm{C})$-poor, Giemsa-dark chromosome bands. Chromosomes 2 and 4, which contain some of the largest deserts in the genome, provide opportunities to examine possible roles of such regions.

Strikingly, $\mathrm{PCDH}$, a protocadherin gene expressed predominately in the brain and heart (Fig. 1a), and its paralogue PCDH10 (at 4q28.3), are each flanked on both sides by unusually large deserts (5.2 $\mathrm{Mb}$ and $3.5 \mathrm{Mb}$ for $P C D H 7$, and $5.1 \mathrm{Mb}$ and $4.0 \mathrm{Mb}$ for $P C D H 10)$. The overall genome architecture of these gene deserts, including the flanking genes, is conserved in mammals and birds (data not shown), but the deserts themselves show only average levels of nucleotide conservation with dog (data provided by the Broad Institute), mouse ${ }^{27}$ and chicken ${ }^{36}$. The duplication event that separated the two protocadherins occurred before mammals diverged from fish, but after they diverged from Ciona intestinalis, suggesting that this arrangement has persisted for hundreds of millions of years.

Two other large deserts (Fig. 1b, c) have portions with a higherthan-average level of nucleotide conservation; these are a $4.7 \mathrm{Mb}$ desert at $4 \mathrm{q} 34.3$ and a $3.5 \mathrm{Mb}$ desert upstream of $Z F H X 1 B$ at $2 \mathrm{q} 22.3$. When compared with chicken, we found 168 segments of conserved sequence for the desert at $4 \mathrm{q} 34.3$ ( 36.5 per $\mathrm{Mb}$ ), 246 segments for the desert at 2q22.3 (74.5 per Mb), and 64 segments in the desert downstream of PCDH7 (13.1 per Mb). Within these intervals, the conserved segments are not uniformly distributed. For the $4 \mathrm{q} 34.3$ desert, the pattern of conservation is similar to that seen in mouse and dog, but there is relatively little conservation with the pufferfish Takifugu (Fugu) rubripes ${ }^{37}$. In contrast, the region upstream of ZFHX1B is enriched in conserved segments in T. rubripes as well as in $\operatorname{dog}$ and mouse. The $Z F H X 1 B$ gene and its neighbour ARHGAP15 appear to be products of a segmental duplication. Their paralogous copies (TCF8 and ARHGAP12) are found on 10p11.22. The intergenic interval upstream of TCF8 is $335 \mathrm{~kb}$ and shows only weak interspecies conservation. There is also little evidence for conservation between the paralogues outside of the coding regions, other than a weak alignment in the $3^{\prime}$-UTRs of ZFHX1B and TCF8. This UTR region is almost 100\% conserved between $Z F H X 1 B$ and the orthologous intervals in chicken and mouse. Thus, the presence of these gene deserts is maintained over longer evolutionary periods than are intervals of high nucleotidelevel conservation within them.

We looked for overrepresented motifs in the conserved noncoding segments in the 2q22.3 region. We evaluated the frequencies of short (4-9 bp) patterns in the conserved and diverged intervals (see Supplementary Methods for details). Using these patterns, we could distinguish between the conserved and non-conserved intervals with $75 \%$ accuracy, suggesting that the conserved regions share short, specific, non-coding (but presumably functional) elements, perhaps transcription factor binding sites, that have been conserved throughout the evolution of mammals and birds.

\section{Recent local duplications-complexity of gene prediction}

The gene for a well-characterized human nuclear export protein, the RAN binding protein 2 ( RanBP2), is contained in a region of recent duplication in the human genome and is in a recombination hot spot on chromosome $2 \mathrm{q}$ (ref. 38). Through a series of rearrangements, this region underwent exon shuffling, domain accretion and deletion, suggesting that this entire region has been extremely dynamic over the last several million years. In a detailed examination of this region, we discovered eight new genes that arose by duplication of RANBP2 (Fig. 2). We called the new gene family $R G P$, for RanBP2-like, GRIP domain-containing proteins. The RGP copies are interspersed in a $9-\mathrm{Mb}$ region on both sides of the centromere, and have significantly modified their gene structure compared with RANBP2. There is experimental evidence for expression of almost all of the $\operatorname{copies}^{39}$, including specific ESTs and cDNAs. Similar duplications are also found in chimpanzee (but other sequenced metazoan genomes, including mouse, have only

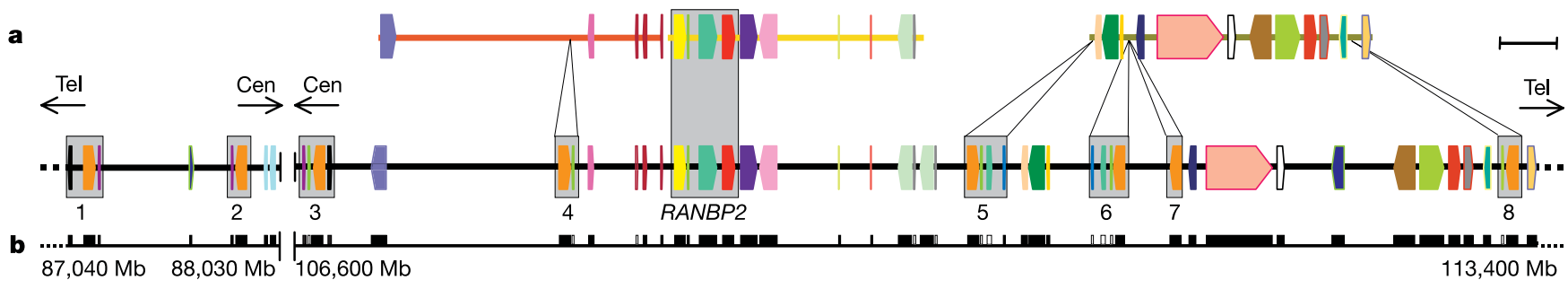

c
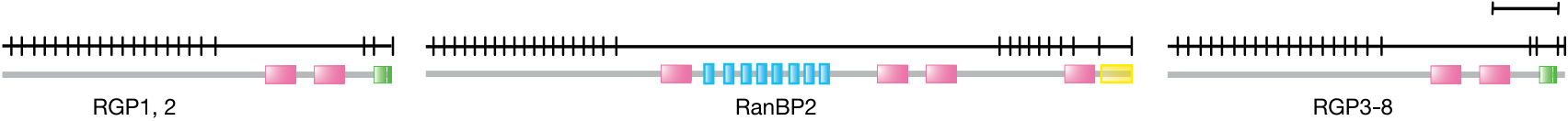

Figure 2 RANBP2 gene duplication in the pericentromeric region of chromosome 2. a, Gene composition. Each coloured bar/arrow represents a different gene; segmentally duplicated regions are highlighted with grey boxes. RANBP2 and its eight paralogues are shown as red and orange arrows, respectively. Regions of orthology in mouse chromosomes 17 (orange), 10 (yellow) and 2 (green) are reported above the human gene bar. Scale bar, $310 \mathrm{~kb}$. b. Filled boxes indicate evidence for expression of genes in a. c, Gene and protein domain architecture of RanBP2 and the RGP family (RGP1-8). Black lines show exon/intron boundaries; pink boxes show Ran binding domains; blue boxes show zinc-finger domains; yellow box shows a cyclophilin A-homologous domain; green boxes show GRIP domains. Scale bar, 300 amino acids. 
one copy). This region provides an excellent example of difficulties encountered during annotation using automated pipelines, and shows how the application of new tools for hunting for gene duplications can reveal interesting evolutionary scenarios.

\section{Segmental duplications}

Segmental duplications of genomic DNA-large, low-copy repeats often spanning hundreds of kilobases-are a prominent feature of the human genome. We performed a detailed analysis of duplicated sequence ( $\geq 90 \%$ sequence identity and $\geq 1 \mathrm{~kb}$ in length), comparing the finished chromosome assemblies to the human genome sequence. Both chromosomes 2 and 4 show less segmental duplication $(4.2 \%$ and $2.3 \%$, respectively) than the genome average (5.2\%) (Supplementary Table 6 and Supplementary Fig. 2). The reduction in segmental duplications on chromosome 4 is especially noticeable within the pericentromeric regions of $4 \mathrm{q} 11-\mathrm{q} 12$, where not a single duplication could be detected within $2 \mathrm{Mb}$ of the centromere. Although it remains possible that additional pericentromeric sequence will be recovered for $4 \mathrm{q} 11-\mathrm{q} 12$, the most proximal segment of sequence does contain $\sim 26 \mathrm{~kb}$ of alpha monomeric satellite DNA, suggesting proximity to the centromere. Given that we found no evidence for duplications using a second detection strategy ${ }^{40}$, and given that fluorescence in situ hybridization (FISH) data $^{41}$ provide little evidence for 4q11-q12 pericentromeric duplications, we suggest that $4 \mathrm{q} 11-\mathrm{q} 12$ has been relatively quiescent in terms of pericentromeric duplications. This is in sharp contrast with $2 \mathrm{p} 11$ and $2 \mathrm{q} 11$, where nearly half of the most proximal $2-\mathrm{Mb}$ pericentromeric region $(899 \mathrm{~kb}$ and $910 \mathrm{~kb}$ respectively) shows extensive recent duplication (Supplementary Fig. 3).

Chromosome 2 is unique to the human lineage of evolution, having emerged as a result of head-to-head fusion of two acrocentric chromosomes that remained separate in other primates. The precise fusion site has been located in 2q13-2q14.1 (ref. 2; hg16:114455823-114455838), where our analysis confirmed the presence of multiple subtelomeric duplications to chromosomes 1, 5, 8, 9, 10, 12, 19, 21 and 22 (Fig. 3; Supplementary Fig. 3a, region A). During the formation of human chromosome 2, one of the two centromeres became inactivated $(2 \mathrm{q} 21$, which corresponds to the centromere from chimp chromosome 13) and the centromeric structure quickly deterioriated ${ }^{42}$. A search of genome sequence for the presence of vestigial centromere and pericentromeric sequences identified a $2.6-\mathrm{Mb}$ region in $2 \mathrm{q} 21.1-2 \mathrm{q} 21.2$ that is enriched for pericentromeric duplications to chromosomes 1, 7, 9, $10,13,14,15,18,21$ and 22 as well as a variety of centromeric satellite repeat sequence motifs (HSAT5, GSATII, ACRO1). The degree of sequence identity of the interchromosomal duplications $(<98 \%)$ suggests that these pericentromeric segmental duplications existed before the formation of this chromosome. Within this 2.6-Mb interval, we identified a relatively large tract of satellite sequence (three tracts totalling $31,198 \mathrm{bp}$ of alpha-satellite sequence over 36,696 bp), which likely demarcates the position of the ancestral centromere (Supplementary Fig. 3a, region B). These data raise the possibility that ancestral telomeres and ancestral centromeres that have disappeared over the course of mammalian chromosomal evolution might be marked by the presence of an abundance of residual pericentromeric and subtelomeric duplications.

By analogy, an interstitial 1.1-Mb region of subtelomeric duplications was identified within 4q26 (Supplementary Fig. 3b, region $\mathrm{D}$ ). This region probably represents a genomic segment that became duplicately transposed to a subtelomeric region and was subsequently dispersed throughout the human genome by secondary subtelomeric-subtelomeric duplications. The average percentage identity between this region and the subtelomeric regions is $97.4 \%$, and among these subtelomeric regions it is $99.0 \%$, indicating more recent duplications or gene conversion events. Although there is not conclusive evidence that this
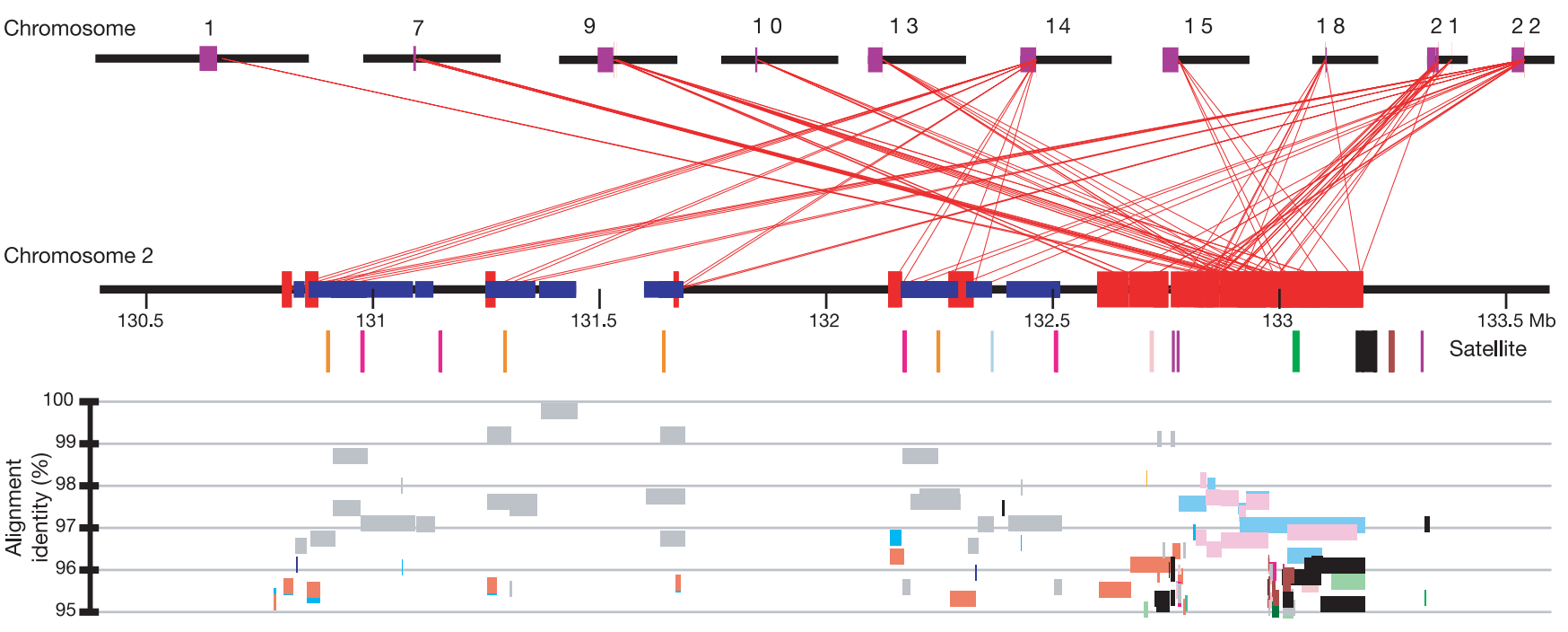

\begin{tabular}{|c|c|c|c|c|c|c|c|}
\hline $\begin{array}{l}\text { Satellite key } \\
\text { ACRO1 }\end{array}$ & Alpha & CER & GSATII & HSAT5 & HSATI & REP522 & TAR1 \\
\hline Chromo & & & & & & & \\
\hline Chr 1 & Chr 2 & Chr 7 & Chr 9 & Chr 10 & Chr 13 & Chr 14 & Chr 15 \\
\hline Chr 18 & Chr 21 & Chr 22 & & & & & \\
\hline
\end{tabular}

Figure 3 Pattern of recent segmental duplications in the vestigial centromere region on chromosome 2. Large (>10 kb), highly similar (>95\%) intrachromosomal (blue) and interchromosomal (red) segmental duplications are shown for a 3-Mb region along the horizontal line of chromosome 2 , in increments of $0.5 \mathrm{Mb}$. The upper panel shows extensive duplication with pericentromeric regions on other chromosomes. Centromeres are shown in purple. The coloured vertical bars underneath chromosome 2 are satellite sequences. The lower panel shows the percentage identity of each pairwise alignment. Coloured bars represent alignments from different chromosomes. 
represents the site of an ancestral telomere, it has the characteristics of such a region, showing an abundance of subtelomeric duplications to human chromosomes 1-9, 11, 16, 19 and 20, an enrichment of short blocks of orthology between human and mouse, and a breakpoint of conserved orthology with the flanking regions between human and mouse. A second short $(30-\mathrm{kb})$ region in $4 \mathrm{q} 32.3$ also shows a breakpoint in conservation with mouse and a similar pattern of subtelomeric duplications. Sixteen duplications ranging in size from $10-20 \mathrm{~kb}$ and showing remarkably high sequence identity (96-97\%) are distributed among ten subtelomeric regions (chromosomes 1, 2, 5-8, 10, 16, 19 and 20, Supplementary Fig. $3 \mathrm{~b}$, region E). In contrast with the region of $4 \mathrm{q} 26$, the organization of this region appears to be conserved in mouse, as revealed by orthologous mouse-human anchor sequences that extend beyond the duplication. Finally, although intrachromosomal duplications are relatively rare on chromosome 4 (Supplementary Figs 4 and 5), one large (750-kb) cluster of tandem intrachromosomal duplication was noted in $4 \mathrm{q} 13.2$. This region contains at least five members of a family of tandemly duplicated microsomal UDPglycosyltransferase genes that are thought to be important in drug detoxification.

\section{Sequence variation}

In the process of constructing contiguous chromosomal sequences from the underlying BAC clone sequences, we encountered several candidate overlaps that showed unusually high levels of variation. For 53 overlaps, totalling $3.1 \mathrm{Mb}$ (with an average difference of $\sim 4$ differences per $\mathrm{kb}$ ), we showed by segregation analysis ${ }^{8}$ that these represented true overlaps, with the differences arising from different alleles of the same locus and not from distinct regions produced by segmental duplication ${ }^{21}$. Extending this analysis to 2,718 overlap regions on chromosomes 2,4 and 7 with at least $5 \mathrm{~kb}$ of overlap, we identified 678 regions with at least three 'polymorphic events', suggesting that the underlying clones represented different haplotypes $^{43}$. Of greatest interest were 24 overlaps (Supplementary Table $7)$, where multiple $(\geq 3)$ consecutive windows varied by at least two standard deviations from the average, possibly indicating segments showing balancing selection ${ }^{44}$. In one example, a $5-\mathrm{kb}$ segment had 75 polymorphic events, and the neighbouring five windows also had more than 18 ( 2 s.d.) polymorphic events. In 15 cases, the highly variant region was found near a gene, including the known genes CRYPTIC (also known as CFC1), TSSC1, LRP1B and GYPE. We sampled across eight of these highly variant regions from the panel of 24 individuals ${ }^{21}$, and confirmed both that these segments were polymorphic in the population and that these regions of extreme variation appear to arise from two distinct haplotype blocks. Further, chimp single nucleotide polymorphisms (SNPs) mapped to the human genome (Chimpanzee GSC, unpublished data) show increased frequencies of polymorphisms (at least four consecutive 5 - $\mathrm{kb}$ windows containing more than mean +2 s.d.) in three of the five regions where orthologous chimpanzee sequence was available. Based on these data, it is unlikely that this represents a locally high mutation rate. Instead, these regions might represent unusually deep coalescents, perhaps as a result of diversifying selection.

\section{Summary and conclusions}

The achievement of highly accurate comprehensive sequence for chromosomes 2 and 4 (and other published human chromosomes) represents a critical step for the Human Genome Project in that it makes possible more detailed and conclusive analyses. On chromosome 2, the local region surrounding the ancestral chromosomal fusion site on 2q13-2q14.1 had previously been described ${ }^{2}$. Here we identified a 2.6-Mb region within $2 \mathrm{q} 21.1-2 \mathrm{q} 21.2$ that is enriched for pericentromeric duplications and centromeric satellite repeat sequence motifs, including a stretch of alpha-satellite sequence that probably identifies the location of the ancestral centromere.
Our results suggest that the abundance of residual pericentromeric and subtelomeric duplications in the human genome, coupled with breakpoints in orthology with mouse, might be markers of ancestral telomeres and centromeres, perhaps representative of recurrent chromosome rearrangements in the human lineage. Also, using orthologous relationships with other genome sequences such as mouse and rat, we have more confidently identified and discriminated between genes and pseudogenes, with accurate classification regarding processed and non-processed pseudogenes. Although the expanding mRNA sets still require cautious evaluation, we have used them to identify a possible human/chimpanzee-specific protein and to characterize a small subset of proteins that contain polymorphisms leading to altered proteins in the human lineage. The sequence data for chromosomes 2 and 4, together with the high-quality annotation of genes and an analysis of homologous regions of vertebrate genomes, reveal a high-level structure consisting of two gene deserts separated by a protocadherin gene. This high-level structure appears to be more stable over evolutionary time than does the low-level nucleotide conservation in other deserts. A genome-wide search revealed a general property of the cadherin gene family: it includes $12 \%$ of all human genes that have a desert immediately on each side, but contains only $0.4 \%$ of all human genes. We have also described genomic segments (on the order of tens of kilobases) for which there are sharp rises in the density of human variation that seem to correlate with haplotype blocks (for example, ref. 43). Although these regions might still represent chance preservation of two alternate haplotype blocks over evolutionary time, they might well also be the result of balancing selection in these regions. It will be important to continue evaluating additional regions, their possible roles in human variation and their correlations with human phenotypes. Critically, immediate access to this sequence will allow researchers in all disciplines to contribute to the understanding of the genome and its relevance in human health.

\section{Methods}

\section{Sequencing in other primates}

We attempted to amplify across each exon in each gene of interest from the following primates: Homo sapiens, Celebes crested macaca (Macaca nigra), Sumatran orangutan (Pongo pygmaeus), Gorilla gorilla, black-handed spider monkey (New World monkey Ateles geoffroyi) and chimpanzee (Pan troglodytes). Primers were chosen in highly conserved human/chimp intronic regions directly flanking the exons. Multiple primers were chosen to increase the possibility of getting a successful product. When we were not able to amplify a given exon in a primate, new primers were chosen on the basis of sequence conservation data from other monkeys where amplification had been successful.

\section{Assessing human deletions/polymorphisms}

A list of possible deletions or polymorphisms in human chromosomes 2 and 4 was created by placement of the fosmid end sequences against the human genome ${ }^{1}$. All fosmid end placements separated by less than 3.5 standard deviations from the average were flagged as possible deletions. Using those criteria, 27 possible deletion regions were flagged (Supplementary Tables 8 and 9). Almost $70 \%$ of these regions across the human genome were found to be polymorphic when tested using PCR analysis ${ }^{1}$. We aligned both of the publicly available Pan troglodytes assemblies (Chimpanzee GSC, unpublished data) against the human genome and manually reviewed the alignments to see whether the possible deletions in human were confirmed by chimpanzee coverage. In 11 cases (41\%), there was good coverage of the entire region in the chimpanzee assembly and there was no suggestion of missing data in the human genome. In 12 cases (44\%), there was additional chimpanzee data throughout the region, suggesting a possible deletion (polymorphic or real) in the human sequence. In one case, there was no chimp coverage of the human region, and in three cases the chimpanzee assembly was too fragmented to determine whether a deletion was present. It remains to be determined whether the deletion in the human sequence was an error or polymorphic.

\section{Gene deserts}

For each panel of Fig. 1, we identified orthologous intervals in the current (as of July 2004) dog, mouse and chicken genome assemblies. After masking known coding regions in the human sequence, we performed alignments to the other three species using the BlastZ program ${ }^{45}$. For the human-chicken alignment, default alignment-scoring parameters were used. For human-dog alignment, we used the following scores: match $=100$; transition $=-500$; transversion $=-1,200$; gap-open $=-2,000$; and gap-extension $=-500$. For human-mouse the respective parameters were $100,-200$, $-600,-1,000$ and -200 . Those parameter values were chosen so that the genome-wide 
fraction of aligned bases was about 0.02 for each species; segments with a higher fraction of aligned bases were considered to be enriched for interspecies conservation. Figure 1 shows the fraction of the segment contained within a local alignment, for non-overlapping $50-\mathrm{kb}$ windows.

Classification of conserved versus nonconserved segments of the human gene desert in Fig. 1c was performed as follows. The gene desert was aligned to the orthologous chicken region, and human intervals showing strong conservation (using an arbitrary threshold of at least $70 \%$ identity with chicken over 100 bp of sequence) or no conservation with chicken were selected. To ensure equal training-set size and length distribution, a subset of the unaligned intervals was prepared by repeatedly randomly selecting an element from the strongly aligned set, and then randomly selecting a piece of an interval from the unaligned set of the same size.

Classification was first performed using the Markov model method described in ref. 46 using only the human sequence (rather than an alignment) as input and the four possible nucleotides as an alphabet. Leave-one-out cross-validation was performed for each pair of training sets at orders 1 through 8 (that is, nucleotide words of 2-9 bp) to determine the best classifying model.

The alternative classification approach constructed a vector of the frequency of occurrence of each possible word for each interval. A support vector machine (SVM) as implemented in LIBSVM ${ }^{47}$ was used to classify each pair of training sets. A gaussian kerne was used and parameters were selected using a grid search and fivefold cross-validation. Because the grid search is more expensive, only word sizes 4-6 were tested.

For comparison, we applied the same methods to sets of RefSeq-annotated $3^{\prime}$ - and $5^{\prime}$-UTRs with similar $(\mathrm{G}+\mathrm{C})$ content and length distributions. The $3^{\prime}$ - and $5^{\prime}$-UTR sets were chosen by selecting the subset of all RefSeq-annotated UTRs with $(\mathrm{G}+\mathrm{C})$ content between 0.36 and 0.37 (similar to the gene desert) and length between 20 and $400 \mathrm{bp}$ (resulting in a similar length distribution). We were able to distinguish them with $67 \%$ accuracy. This suggests that the human sequence of the strongly conserved and unconserved intervals in this gene desert show at least as much difference in characteristic short patterns used by the classification schemes as do $3^{\prime}$ - and $5^{\prime}$-UTRs.

Received 25 October 2004; accepted 11 February 2005; doi:10.1038/nature03466.

1. Human Genome Sequencing Consortium. Finishing the euchromatic sequence of the human genome. Nature 431, 931-945 (2004)

2. Fan, Y., Linardopoulou, E., Friedman, C., Williams, E. \& Trask, B. J. Genomic structure and evolution of the ancestral chromosome fusion site in $2 \mathrm{q} 13-2 \mathrm{q} 14.1$ and paralogous regions on other human chromosomes. Genome Res. 12, 1651-1662 (2002).

3. International Human Genome Sequencing Consortium. Initial sequencing and analysis of the human genome. Nature 409, 860-921 (2001).

4. The International Human Genome Mapping Consortium. A physical map of the human genome. Nature 409, 934-941 (2001).

5. Felsenfeld, A., Peterson, J., Schloss, J. \& Guyer, M. Assessing the quality of the DNA sequence from the Human Genome Project. Genome Res. 9, 1-4 (1999).

6. Schmutz, J., Wheeler, J., Grimwood, J., Dickson, M. \& Myers, R. M. Assessing the quality of finished genomic sequence. Cold Spring Harb. Symp. Quant. Biol. 68, 31-37 (2003).

7. Riethman, H. C. et al. Integration of telomere sequences with the draft human genome sequence. Nature 409, 948-951 (2001).

8. Hillier, L. W. et al. The DNA sequence of human chromosome 7. Nature 424, 157-164 (2003).

9. The International HapMap Consortium. The International HapMap Project. Nature 426, 789-796 (2003).

10. Dib, C. et al. A comprehensive genetic map of the human genome based on 5,264 microsatellites. Nature 380, 152-154 (1996)

11. Broman, K. W., Murray, J. C., Sheffield, V. C., White, R. L. \& Weber, J. L. Comprehensive human genetic maps: individual and sex-specific variation in recombination. Am. J. Hum. Genet. 63, 861-869 (1998).

12. Kong, A. et al. A high-resolution recombination map of the human genome. Nature Genet. 31, 241-247 (2002).

13. Schuler, G. D. et al. A gene map of the human genome. Science 274, 540-546 (1996)

14. Olivier, M. et al. A high-resolution radiation hybrid map of the human genome draft sequence. Science 291, 1298-1302 (2001).

15. Antequera, F. \& Bird, A. Number of CpG islands and genes in human and mouse. Proc. Natl Acad. Sci. USA 90, 11995-11999 (1993).

16. Wang, Y. \& Leung, F. C. An evaluation of a new criteria for $\mathrm{CpG}$ islands in the human genome. Bioinformatics 20, 1170-1177 (2004).

17. Liu, C. X., Musco, S., Lisitsina, N. M., Yaklichkin, S. Y. \& Lisitsyn, N. A. Genomic organization of a new candidate tumor suppressor gene, LRP1B. Genomics 69, 271-274 (2000).

18. Chan, A. C. et al. ZAP-70 deficiency in an autosomal recessive form of severe combined immunodeficiency. Science 264, 1559-1601 (1994).

19. Pruitt, K. D. \& Maglott, D. R. RefSeq and LocusLink: NCBI gene-centered resources. Nucleic Acids Res 29, 137-140 (2001).

20. Mammalian Gene Collection Program Team. Generation and initial analysis of more than 15,000 full-length human and mouse cDNA sequences. Proc. Natl Acad. Sci. USA 99, 16899-16903 (2002).

21. Sachidanandam, R. et al. A map of human genome sequence variation containing 1.42 million single nucleotide polymorphisms. Nature 409, 928-933 (2001).

22. Nicolaides, N. C. et al. Mutations of two PMS homologues in hereditary nonpolyposis colon cancer Nature 371, 75-80 (1994).

23. Birney, E. \& Durbin, R. Using GeneWise in the Drosophila annotation experiment. Genome Res. 10, 547-548 (2000).

24. Korf, I., Flicek, P., Duan, D. \& Brent, M. R. Integrating genomic homology into gene structure prediction. Bioinformatics 17(S1), 140-148 (2001).
25. Suyama, M., Torrents, D. \& Bork, P. BLAST2GENE: a comprehensive conversion of BLAST output into independent genes and gene fragments. Bioinformatics 20, 1968-1970 (2004).

26. Rat Genome Sequencing Project Consortium. Genome sequence of the Brown Norway rat yields insights into mammalian evolution. Nature 428, 493-521 (2004).

27. Mouse Genome Sequencing Consortium. Initial sequencing and comparative analysis of the mouse genome. Nature 420, 520-562 (2002)

28. Torrents, D., Suyama, M., Zdobnov, E. \& Bork, P. A genome-wide survey of human pseudogenes. Genome Res. 13, 2559-2567 (2003).

29. Zhang, Z., Carriero, N. \& Gerstein, M. Comparative analysis of processed pseudogenes in the mouse and human genomes. Trends Genet. 20, 62-67 (2004).

30. Eddy, S. R. Computational genomics of noncoding RNA genes. Cell 109, 137-140 (2002).

31. Mishmar, D., Ruiz-Pesini, E., Brandon, M. \& Wallace, D. C. Mitochondrial DNA-like sequences in the nucleus (NUMTs): Insights into our African origins and the mechanism of foreign DNA integration. Hum. Mutat. 23, 125-133 (2004)

32. Mulder, N. J. et al. InterPro: an integrated documentation resource for protein families, domains and functional sites. Brief. Bioinform. 3, 225-235 (2002).

33. Zdobnov, E. M. \& Apweiler, R. InterProScan-an integration platform for the signature-recognition methods in InterPro. Bioinformatics 17, 847-848 (2001)

34. The Gene Ontology Consortium. Creating the Gene Ontology resource: design and implementation. Genome Res. 11, 1425-1433 (2001).

35. Ovcharenko, I. et al. Evolution and functional classification of vertebrate gene deserts. Genome Res. $15,137-145$ (2005)

36. International Chicken Genome Sequencing Consortium. Sequence and comparative analysis of the chicken genome provide unique perspectives on vertebrate evolution. Nature 432, 695-715 (2004).

37. Aparicio, S. et al. Whole-genome shotgun assembly and analysis of the genome of Fugu rubripes. Science 297, 1301-1310 (2002)

38. Bailey, J. A. et al. Recent segmental duplications in the human genome. Science 297, 1003-1007 (2002).

39. Ciccarelli, F. D. et al. Complex genomic rearrangements lead to novel primate gene function. Genome Res. 15, 343-351 (2005).

40. Bailey, J. A. et al. Human-specific duplication and mosaic transcripts: the recent paralogous structure of chromosome 22. Am. J. Hum. Genet. 70, 83-100 (2002).

41. The BAC Resource Consortium. Integration of cytogenetic landmarks into the draft sequence of the human genome. Nature 409, 953-958 (2001).

42. Yunis, J. J. \& Prakash, O. The origin of man: a chromosomal pictorial legacy. Science 215, 1525-1530 (1982).

43. Gabriel, S. B. et al. The structure of haplotype blocks in the human genome. Science 296, 2225-2229 (2002)

44. Daly, M. J., Rioux, J. D., Schaffner, S. F., Hudson, T. J. \& Lander, E. S. High-resolution haplotype structure in the human genome. Nature Genet. 29, 229-232 (2001).

45. Schwartz, S. et al. Human-mouse alignments with BLASTZ. Genome Res. 13, 103-107 (2003).

46. Kolbe, D. et al. Regulatory potential scores from genome-wide three-way alignments of human, mouse, and rat. Genome Res. 14, 700-707 (2004)

47. Chang, C. C. \& Chih-Jen, L. LIBSVM: a library for support vector machines. Software at 〈http:// www.csie.ntu.edu.tw/ cjlin/libsvm/ $>$ (2001).

48. Roest-Crollius, H. et al. Estimate of human gene number provided by genome-wide analysis using Tetraodon nigroviridis DNA sequence. Nature Genet. 25, 235-238 (2000).

49. Dehal, P. et al. The draft genome of Ciona intestinalis: insights into chordate and vertebrate origins. Science 298, 2157-2167 (2002)

50. Kent, W. J. BLAT-the BLAST-like alignment tool. Genome Res. 12, 656-664 (2002).

Supplementary Information accompanies the paper on www.nature.com/nature.

Acknowledgements The authors would like to thank the staff, past and present, of the Washington University Genome Sequencing Center as well as the following laboratories that contributed sequence segments to the final chromosome 2 and 4 sequence: the Broad Institute of MIT; Stanford DNA Sequencing and Technology Development Center; the Sanger Centre; National Yang-Ming University in Taipei; Genoscope; Baylor College of Medicine; the Joint Genome Institute; University of Washington Multimegabase Sequencing Center; DOE Joint Genome Institute; and Roswell Park Cancer Institute. We thank T. Hubbard, J. Ashurst, J. Gilbert and S. Keenan for Ensembl pipeline assistance. We thank the Broad Institute of MIT, Harvard and Agencourt Bioscience for making a preliminary assembly of dog genome sequence available to us. We thank the Zebrafish Sequencing Group at the Sanger Centre for the Danio rerio preliminary protein set. We thank the International HapMap Consortium and their sponsors for use of their data and J. Mullikin for help with alignment of their data to the human genome. We thank the Chimpanzee Genome Sequencing Consortium for use of the chimpanzee sequence data. The authors would also like to acknowledge the efforts of the HUGO Gene Nomenclature Committee. Finally, the authors acknowledge the National Human Genome Research Institute for funding this work.

Competing interests statement The authors declare that they have no competing financial interests.

Correspondence and requests for materials should be addressed to R.K.W.

(rwilson@watson.wustl.edu). All reported DNA sequences have been deposited in GenBank or EMBL. Accession numbers for the chromosome sequence analysed for this paper can be found in Supplementary Table 1. The updated chromosome 2 and 4 sequences can be accessed through GenBank accession numbers NC_000002 (chromosome 2) and NC_000004 (chromosome 4). Primate resequencing data can be accessed using GenBank accession numbers CZ179368-CZ179565. The mRNA resequencing data can be accessed via GenBank/dbSNP identifiers ss35032449-ss35032461, ss35033273-ss35033317. 